\title{
Correction to: Preoperative Neutrophil-to-Lymphocyte Ratio Level is a Predictor of Postoperative Fertility in Infertile Patients with Ovarian Endometrioma
}

\author{
Lizhen $\operatorname{Lin}^{1,2} \cdot$ Guan Lin ${ }^{1,2} \cdot$ Huixin Lian ${ }^{1,2} \cdot$ Qingshan Chen ${ }^{1,2} \cdot$ Penghui Huang ${ }^{1,2} \cdot$ Shunhe Lin ${ }^{1,2} \cdot$ Zhenhong Wang $^{1,2}$. \\ Jun Shi ${ }^{1,2} \cdot$ Chaobin $\mathrm{Liu}^{1,2} \cdot \mathrm{Xi} \mathrm{Xie}^{1,2}$ (1) \\ Published online: 4 February 2022 \\ (c) Society for Reproductive Investigation 2022
}

Correction to: Reprod. Sci.

https://doi.org/10.1007/s43032-021-00782-1

This article was updated to correct the National Nature Science Foundation of China grant number in the Funding section.

Lizhen Lin and Guan Lin contributed equally to this work.

The original article can be found online at https://doi.org/10.1007/ s43032-021-00782-1.

\section{Chaobin Liu}

1cb7073@163.com

$\triangle$ Xi Xie

xixie1117@163.com

1 Department of Gynecology and ObstetricsFujian Maternity and Child Health Hospital, Afliated Hospital of Fujian Medical University, Fuzhou, China

2 Fujian Key Laboratory of Women and Children's Critical Diseases Research, Fujian Maternity and Child Health Hospital, Afliated Hospital of Fujian Medical University, Fuzhou, China 